\title{
Imagen mental y personalidad en equipos de trabajo de estudiantes universitarios.
}

\section{Mental image and personality in university students' work teams}

\author{
Mario Lado, Adolfo Pena \\ Universidade de Santiago de Compostela
}

\begin{abstract}
Resumen
El objetivo de este estudio es describir, a través de las imágenes mentales, el perfil de personalidad de los integrantes de equipos de trabajo y conocer la influencia de variables demográficas. Recientes meta-análisis han mostrado que la Conciencia, perteneciente al modelo de los cinco factores de personalidad, es un buen predictor del desempeño académico. Existe escasa literatura acerca de la imagen mental como proceso mental para describir el perfil de personalidad. 226 estudiantes de Grado universitario participaron en el estudio y los resultados indicaron que los adjetivos relacionados con el factor de Conciencia y Amigabilidad eran los más frecuentes. No existen diferencias significativas en las variables demográficas utilizadas en este estudio, excepto la variable del género.
\end{abstract}

Palabras clave: Imagen mental, personalidad, equipos de trabajo.

\begin{abstract}
The objective of this study is to describe, through the mental images, the personality profile of the members of work teams and to know the influence of demographic variables. Recent meta-analyses have shown that Consciousness, pertaining to the model of the five personality factors, is a good predictor of academic performance. There is little literature about the mental image as a mental process to describe the personality profile. 226 university students participated in the study and the results indicated that the adjectives related to Consciousness and Agreeableness factors were the most frequent. There are no significant differences in the demographic variables used in this study, except the gender variable.

Key words: mental image, personality, team work.
\end{abstract}

\section{Introducción}

El objetivo de este estudio es analizar y describir, a través de las imágenes mentales de los propios integrantes de los equipos de trabajo, las características de personalidad más relevantes que definen a una persona y describen su comportamiento cuando interactúa en dichos equipos de trabajo, así como conocer la posible influencia de diversas características demográficas en los resultados.

Existe escasa literatura acerca de la imagen mental como proceso mental para analizar y describir las características de personalidad en los individuos y su comportamiento en los equipos de trabajo; por otra parte, existe abundante literatura acerca de las dimensiones de personalidad y su relación con el desempeño, tanto en el ámbito laboral como académico. Recientes meta-análisis acerca de la validez de las dimensiones de personalidad han mostrado que el factor de Conciencia es un buen predictor del desempeño (Barrick \& Mount, 1991; Poropat, 2009; Salgado, 1997). Para la descripción de la personalidad de los individuos se han desarrollado inventarios y cuestionarios de autoinforme basados en los diferentes modelos de personalidad (Cattell, 1943; Eysenck, 1963; Norman, 1963); además, otro método de descripción corresponde al modelo utilizado por Peres and Garcia (1962) con el fin de analizar los adjetivos y descriptores del comportamiento presenten en diversas declaraciones que unas personas habían hecho sobre otras en diversos procedimientos de selección de personal, promoción, acceso e organizaciones, etc. El procedimiento utilizado por Peres y García (1962) ha sido replicado posteriormente en otros estudios por Aamodt, Bryan y Whitcomb $(1989,1993)$ y Aamodt (2006), para identificar los adjetivos descriptores de comportamientos y evaluar, así, el contenido de las cartas de recomendación en diversos procesos de selección de personal. De esa manera, utilizando este procedimiento se pretende analizar los adjetivos de cada cuestionario aportado por cada participante y señalar los rasgos de personalidad relacionados con cada adjetivo.

Desde el principio de los tiempos las personas ya han venido trabajando en equipo, con mayor o menor éxito con diversos fines, desde la mera subsistencia, seguridad, hasta emprender tareas complejas, investigaciones, descubrimientos, etc.

La mayor parte de las organizaciones reconocen la importancia del trabajo en equipo no sólo como un medio para mejorar su competitividad, sino como un sistema de organización del trabajo que permita mejorar el clima laboral, la comunicación interna, la integración de los nuevos miembros, la transmisión de sus valores y cultura, etc. (Vadillo, 2013).

Trabajar en equipo es colaborar organizadamente para la obtención de un objetivo común. De ello se extrae que cada individuo tiene una personalidad concreta, unas 
habilidades, conocimientos y experiencias específicas, que se diferencian de las del resto de miembros del equipo, para ser utilizadas en aras de la obtención de un objetivo común. Todas estas características han de relacionarse y coordinarse ya que todas son importantes para la eficacia del equipo.

La personalidad es un conjunto de características o rasgos que definen a una persona, es decir, los pensamientos, sentimientos, actitudes, hábitos y la conducta de cada individuo que de manera muy particular hacen que las personas sean diferentes a las demás. Estos rasgos o características son utilizados para definirse a sí mismo o para definir a otra persona, están enraizados en el lenguaje humano, estables en el tiempo y a través de las situaciones, están relacionados con la conducta humana y se agrupan en dimensiones de personalidad. La literatura incluye varios modelos e instrumentos para evaluar los diferentes rasgos y dimensiones de personalidad; por ejemplo, el modelo de Cattell (1943) consta de 187 elementos agrupados en 16 factores de primer orden, así como cuatro puntuaciones de factores de segundo orden; el modelo propuesto por Eysenck (1963) incluye las dimensiones de psicoticismo, extraversión y neuroticismo; el modelo de los cinco grandes factores (Norman, 1963) que distingue cinco dimensiones: estabilidad emocional, extroversión, apertura a la experiencia, amigabilidad y conciencia; etc.

\section{Método}

El objeto de este trabajo es conocer, a través de los propios integrantes de los equipos de trabajo, cuales son las características de personalidad más relevantes de los compañeros en un equipo de trabajo, conocer si existen otras características personales relevantes, así como, si las variables demográficas influyen en estas características.

\section{Muestra}

Participaron un total de 228 alumnos (72 varones y 154 mujeres), con un rango de edad entre los 18 y 52 años $(M=22.41, S D=5.84)$. Por cursos, 77 alumnos estaban matriculados en $1^{\circ}$ curso, 59 en $2^{\circ}, 38$ en $3^{\circ}$ y 52 en $4^{\circ}$ curso de Grado de Relaciones Laborales y recursos Humanos (USC).

\section{Instrumentos}

Se utilizó un cuestionario elaborado para este estudio que incluía cuatro preguntas cerradas y una abierta, además de las instrucciones y de los datos biográficos correspondientes al sexo, edad, curso y calificación de acceso a la universidad. Las cuatro preguntas abiertas eran las siguientes: (1) ¿cuáles son las características del mejor compañero/a de equipo que hayas tenido?, (2) ¿cuáles son las características del peor compañero/a de equipo que hayas tenido?, (3) ¿cuáles serían las características del compañero/a ideal de equipo?, y (4) ¿cuáles son las características que mejor te describen como compañero/a de equipo? La pregunta cerrada trata sobre las características imprescindibles (I), deseables pero no imprescindibles (D) o no necesarias (NN) que tienen relación con el compañero perfecto. Está compuesta por un total de 18 características divididas en
6 grupos (Estabilidad Emocional, Amigabilidad, Conciencia, Extraversión, Apertura a la Experiencia y Habilidades) con 3 características cada uno. Se responde escribiendo I, D o $\mathrm{NN}$ al lado derecho de cada característica.

\section{Procedimiento}

Se administró un cuestionario a los alumnos en diversas sesiones interactivas, previa autorización de los profesores. A la entrega de los cuestionarios se les explicaba a los alumnos las partes del cuestionario y se les indicaba que la participación era voluntaria y que podían salir durante los 15 minutos que iba a durar el acto. Todos los alumnos aceptaron participar.

Posteriormente a la realización de las encuestas, se procedió a la recogida de datos, codificación y análisis con el programa SPSS 20.

Se recogieron todos los adjetivos utilizados por los participantes y en las cuatro categorías (el mejor, el peor, el ideal y la auto-descripción). Cada uno de esos adjetivos fue clasificado en función del factor de personalidad que le correspondía: por ejemplo, el adjetivo "ordenado" se contabilizaba como un adjetivo perteneciente al factor de "Conciencia", perteneciente al modelo de los Cinco Grandes factores de Personalidad.

\section{Resultados}

El tratamiento de datos se realizó a través de varios estadísticos: las frecuencias con que se mencionaban de las características de personalidad, y los análisis de varianza (ANOVA) para analizar la influencia de cada variable demográfica sobre las características de personalidad.

En cuanto a las frecuencias de los adjetivos los resultados indicaron que, para cualquier categoría, los participantes utilizan, con mayor frecuencia, adjetivos relacionados con los factores de personalidad "Conciencia" y "Amigabilidad" para definir las características relevantes de los miembros de un equipo de trabajo. Esta diferencia es notable ya que la frecuencia de los adjetivos referidos al factor de conciencia duplica a la frecuencia de los adjetivos referidos a "amigabilidad" y, éste, a su vez, a los demás factores de personalidad. En la Tabla 1 se muestran las frecuencias de los adjetivos aportados por los participantes. 
Tabla 1.

Frecuencias de adjetivos por categorías y por factores de personalidad.

\begin{tabular}{lrrrrc}
\hline Categoría & E.E. & EX & AP & AM & CO \\
\hline Mejor compañero & 41 & 74 & 105 & $\mathbf{1 8 2}$ & $\mathbf{4 7 1}$ \\
Peor compañero & 53 & 103 & 44 & $\mathbf{1 2 6}$ & $\mathbf{5 1 2}$ \\
Compañero Ideal & 64 & 123 & 139 & $\mathbf{2 6 0}$ & $\mathbf{5 0 9}$ \\
Auto-descripción & 96 & 109 & 110 & $\mathbf{3 3 7}$ & $\mathbf{4 3 9}$ \\
Total & 254 & 409 & 398 & $\mathbf{9 0 5}$ & $\mathbf{1 9 3 1}$
\end{tabular}

Nota EE=Estabilidad emocional; EX=Extroversión; $\mathrm{AP}=$ Apertura a la experiencia; $\mathrm{AM}=$ Amigabilidad $\mathrm{CO}=$ Conciencia.

Se elaboró un segundo análisis paralelo al anterior a través de una pregunta estructurada en el cuestionario. En este apartado se mostraban diversos calificativos relacionados con factores de personalidad y se les pedían a los participantes que les adjudicaran un valor determinado a cada uno: si ese calificativo les parecía imprescindible para la categoría de compañero/a (3 puntos), si les parecía deseable aunque no imprescindible (2 puntos), y si ese calificativo no era ni imprescindible ni necesario (1 punto).

Los resultados indican, una vez más, y a través de otro método de análisis, que los factores por los que los participantes mostraban más tendencia para describir la categoría de "compañero/a" son aquellos referidos a los factores de Conciencia y Amigabilidad. Los resultados se muestran en la Tabla 2.

Tabla 2.

Valoración de los adjetivos relacionados con los factores de personalidad.

\begin{tabular}{llllll}
\hline Factor & E.E. & EX & AP & AM & CO \\
\hline Puntos & 1360 & 1319 & 1268 & 1757 & 1771
\end{tabular}

Nota $:$ EE=Estabilidad emocional; $\mathrm{EX}=$ Extroversión; $\mathrm{AP}=$ Apertura a la experiencia; $\mathrm{AM}=$ Amigabilidad $\mathrm{CO}=$ Conciencia.

El tercer análisis de este estudio consistía en averiguar si las variables demográficas (sexo, edad, curso, calificación PAAU) influían en la adjudicación de los adjetivos relacionados con los factores de personalidad. En primer lugar, se elaboró un ANOVA para conocer si el sexo de los participantes influía en la cantidad de descriptores que aportaba en sus relatos.

Los resultados de la primera variable (sexo) indicaron que las mujeres utilizaban, como promedio, más adjetivos de extroversión que los varones $(M=5.99$ vs $M=5.51)$, y el estadístico de significatividad $(\mathrm{p}<.01)$ indicaba que esta diferencia es significativa. De manera similar ocurre con los adjetivos relacionados con el factor de conciencia $(M=7.95$ vs $M=7.60)$, y el estadístico de significatividad $(\mathrm{p}<.01)$ indicaba también que esta diferencia es significativa. Por tanto, los resultados indicaron que el sexo influye en la adjudicación de adjetivos relacionados con los factores de personalidad extroversión y conciencia. Los datos de los resultados se muestran en la Tabla 3 .

Tabla 3.

Resultados de la influencia del Sexo sobre la frecuencia de los rasgos de personalidad.

\begin{tabular}{|c|c|c|c|c|c|c|}
\hline \multirow[b]{2}{*}{ Factores } & \multicolumn{2}{|c|}{ Varón } & \multicolumn{2}{|c|}{ Mujer } & \multirow[b]{2}{*}{$F$} & \multirow[b]{2}{*}{$p$} \\
\hline & $M$ & $S D$ & $M$ & $S D$ & & \\
\hline Estabilidad E & 5.97 & 1.074 & 6.03 & 1.193 & .10 & .745 \\
\hline Extroversión & 5.51 & 1.138 & 5.99 & 1.204 & 7.83 & $.006 * *$ \\
\hline Apertura Exp. & 5.51 & 1.048 & 5.66 & 1.122 & .81 & .367 \\
\hline Amigabilidad & 7.63 & .971 & 7.84 & 1.017 & 2.34 & .127 \\
\hline Conciencia & 7.60 & .799 & 7.95 & .927 & 7.64 & $.006 * *$ \\
\hline
\end{tabular}

$(* *) p<.01$

Con respecto a la variable "edad" los resultados indicaron que, aunque existen diferencias a través de los grupos de edad en la adjudicación de adjetivos, estas diferencias no son significativas en ningún factor. Por tanto los resultados indican que la edad no influye en la adjudicación de adjetivos relacionados con los factores de personalidad. Los datos de los resultados se muestran en la Tabla 4.

Tabla 4

Resultados de la influencia de la edad sobre la frecuencia de los rasgos de personalidad.

\begin{tabular}{|c|c|c|c|c|c|c|}
\hline \multirow[b]{2}{*}{ Factores } & \multicolumn{2}{|c|}{$>$ Media } & \multicolumn{2}{|c|}{$<$ Media } & \multirow[b]{2}{*}{$F$} & \multirow[b]{2}{*}{$p$} \\
\hline & $M$ & $S D$ & $M$ & $S D$ & & \\
\hline Est. Emocional & 15.91 & 1.18 & 6.04 & 1.15 & .53 & .465 \\
\hline Extroversión & 5.88 & 1.38 & 5.82 & 1.14 & .077 & .782 \\
\hline Apertura Exp. & 5.68 & 1.06 & 5.59 & 1.11 & .284 & .595 \\
\hline Amigabilidad & 7.75 & .90 & 7.78 & 1.04 & .043 & .835 \\
\hline Conciencia & 7.89 & .97 & 7.82 & .88 & .292 & .589 \\
\hline
\end{tabular}

Los resultados de la variable "Curso" indicaron que, a medida que avanzan los cursos, los participantes incrementan el promedio de adjetivos referidos a los factores de personalidad extroversión y apertura a la experiencia (p.ej., Medias de 6.45 y 5.81 frente a 5.60 y 5.78 en el factor de extroversión; y Media de 6.16 y 5.63 frente a 5.34 y 5.59 en el factor de apertura). Estas diferencias en estos dos factores es significativa ( $p=.004$ y $p=.002$, respectivamente). Las diferencias existentes en los promedios de adjetivos referidos a los demás factores de personalidad no son significativas.

Por tanto, los resultados indicaron que el curso al que pertenecen los participantes de este estudio influye en la adjudicación de adjetivos relacionados con los factores de personalidad extroversión y apertura a la experiencia. Los datos de los resultados se muestran en la Tabla 5. 
Tabla 5.

Resultados de la influencia del curso sobre la frecuencia de los rasgos de personalidad.

\begin{tabular}{|c|c|c|c|c|c|c|}
\hline \multirow[b]{2}{*}{ Factores } & \multicolumn{2}{|c|}{$1^{\circ}$ curso } & \multicolumn{2}{|c|}{$2^{\circ}$ curso } & \multicolumn{2}{|c|}{$3^{\circ}$ curso } \\
\hline & $M$ & $S D$ & $M$ & $S D$ & $M$ & $S D$ \\
\hline Est. Emociona & 15.79 & 1.29 & 6.08 & 1.07 & 6.21 & 1.23 \\
\hline Extroversión & 5.60 & 1.08 & 5.78 & .97 & 6.45 & 1.57 \\
\hline Apertura Exp. & 5.34 & 1.11 & 5.59 & 1.04 & 6.16 & 1.18 \\
\hline Amigabilidad & 7.64 & .99 & 7.78 & 1.05 & 7.87 & 1.02 \\
\hline Conciencia & 7.83 & .85 & 7.69 & .92 & 8.08 & .75 \\
\hline
\end{tabular}

Tabla 5. (Continuación)

Resultados de la influencia del curso sobre la frecuencia de los rasgos de personalidad.

\begin{tabular}{|c|c|c|c|c|}
\hline \multirow[b]{2}{*}{ Factores } & \multicolumn{4}{|c|}{$4^{\circ}$ curso } \\
\hline & $M$ & $S D$ & $F$ & $p$ \\
\hline Est. Emocional & 6.10 & .93 & 1.484 & .220 \\
\hline Extroversión & 5.81 & 1.19 & 4.548 & $.004 * *$ \\
\hline Apertura Exp. & 5.63 & .97 & 4.990 & $.002 * *$ \\
\hline Amigabilidad & 7.90 & .96 & .881 & .452 \\
\hline Conciencia & 7.83 & .90 & 1.411 & .240 \\
\hline
\end{tabular}

(**) $p<.01$

Con respecto a la variable "PAAU" los resultados indicaron que, aunque existen diferencias a través de las calificaciones de acceso a la universidad en la adjudicación de adjetivos, estas diferencias no son significativas en ningún factor. Por tanto los resultados indican que la calificación PAAU no influye en la adjudicación de adjetivos relacionados con los factores de personalidad. Los datos de los resultados se muestran en la Tabla 6.

Tabla 6.

Resultados de la influencia de la calificación PAAU sobre la frecuencia de los rasgos de personalidad.

\begin{tabular}{|c|c|c|c|c|c|c|}
\hline \multirow[b]{2}{*}{ Factores } & \multicolumn{2}{|c|}{$>$ Promedio } & \multicolumn{2}{|c|}{$<$ Promedio } & \multirow[b]{2}{*}{$F$} & \multirow[b]{2}{*}{$p$} \\
\hline & $M$ & $S D$ & $M$ & $S D$ & & \\
\hline Est. Emociona & 15.98 & 1.08 & 6.03 & 1.21 & .11 & .741 \\
\hline Extroversión & 5.69 & 1.18 & 5.95 & 1.21 & 2.58 & .110 \\
\hline Apertura Exp. & 5.63 & 1.06 & 5.60 & 1.13 & .03 & .866 \\
\hline Amigabilidad & 7.70 & 1.09 & 7.83 & .94 & .96 & .327 \\
\hline Conciencia & 7.89 & .84 & 7.80 & .84 & .49 & .483 \\
\hline
\end{tabular}

\section{Conclusiones}

El objetivo de este trabajo consistía en conocer el contenido de los adjetivos que un grupo de estudiantes universitarios utilizaban para describir los rasgos de personalidad relevantes para el papel de compañero/a de equipo y, además, conocer si las variables demográficas influían en la frecuencia de utilización de los mismos.

Los resultados de este estudio indicaron que los adjetivos que hacen referencia a los rasgos de personalidad del factor de conciencia (p.ej., aplicado, organizado, cuidadoso, constante, etc.) son los más frecuentemente utilizados por los participantes del estudio, y eso supone corroborar el papel importante del factor de conciencia en la predicción del desempeño.

Los resultados también indican que el sexo de los participantes es la única variable que influye en la frecuencia de los descriptores, en el sentido de que las mujeres utilizan más descriptores relacionados con el factor de conciencia que los varones. Ninguna otra variable influye en este resultado.

Los resultados de este estudio coinciden con otros meta-análisis anteriores en la importancia del factor de conciencia como predictor del desempeño; además, demuestra que los rasgos de personalidad correspondientes a la dimensión de Conciencia están presentes en la mente de manera viva, así como en la verbalización de las declaraciones; por último, estos resultados indican que no existen variables de tipo demográfico que influyen en estos resultados, a excepción de la significativa diferencia de las mujeres sobre los varones en destacar la dimensión de conciencia en las perdonas que participan en un equipo de trabajo.

A partir de los resultados de este estudio se sugiere diversificar la muestra a diversas facultades $\mathrm{y}$, por otra parte, ampliarlo a entornos laborales y analizar la comparación entre ambos contextos.

\section{Referencias}

Aamodt, M. G. (2006). Validity of recommendations and references. Assessment Council News, 4-6.

Aamodt, M. G., Bryan, D. A., \& Whitcomb, A. J. (1989). Validation of the Peres and Garcia Technique for Predicting Performance with Letters of Recommendation. In Proceedings of the Annual Meeting of the International Personnel Management Association, Assessment Council, Orlando (pp. 151-154).

Aamodt, M. G., Bryan, D. A., \& Whitcomb, A. J. (1993). Predicting performance with letters of recommendation. Public Personnel Management, 22(1), 81-90. http://dx.doi.org/10.1177/ 009102609302200106

Barrick, M. R., \& Mount, M. K. (1991). The big five personality dimensions and job performance: a meta-analysis. Personnel psychology, 44(1), 1-26. http://dx.doi.org/10.1111/j.1744-6570.1991.tb00688.x

Cattell, R. B. (1943). The description of personality: basic traits resolved into clusters. The journal of abnormal and social psychology, 38(4), 476. http://dx.doi.org/10.1037/h0054116

Eysenck, H. J. (1963). Biological basis of personality. Nature, 199(4898), 1031-1034. http://dx.doi.org/10.1038/1991031a0

Norman, W. T. (1963). Toward an adequate taxonomy of personality attributes: Replicated factor structure in peer nomination personality ratings. Journal of Abnormal and Social Psychology, 66, 574- 583. http://dx.doi.org/10.1037/h0040291

Peres, S. H., \& Garcia, J. R. (1962). Validity and dimensions of descriptive adjectives used in reference letters for engineering applicants. Personnel 
Psychology, 15(3), 279-286. http://dx.doi.org/10.1111/j.1744-6570.1962.tb01622.x

Poropat, A. E. (2009). A meta-analysis of the five-factor model of personality and academic performance. Psychological bulletin, 135(2), 322. http://dx.doi.org/10.1037/a0014996

Salgado, J. F. (1997). The five factor model of personality and job performance in the European Community. Journal of Applied Psychology, 82(1), 30-43. http://dx.doi.org/10.1037/0021-9010.82.1.30

Vadillo, M. T. P. (2013). Liderazgo y motivación de equipos de trabajo. ESIC Editorial. 